\title{
CLINICAL STUDY AND MANAGEMENT OF PERITONITIS SECONDARY TO HOLLOW VISCOUS PERFORATION
}

\author{
Umapathi P1, Mahesh Babu G²
}

${ }^{1}$ Assistant Professor, Department of General Surgery, Apollo Institute of Medical Sciences and Research and District Government Hospital, Chittoor, Andhra Pradesh, India.

${ }^{2}$ Civil Assistant Surgeon, Department of General Surgery, Apollo Institute of Medical Sciences and Research and District Government Hospital, Chittoor, Andhra Pradesh, India.

\begin{tabular}{l}
\hline ABSTRACT \\
BACKGROUND \\
Hollow viscous perforation causing peritonitis (Secondary) is the most common surgical emergency worldwide. Peptic ulcer \\
perforation is being the most common cause in developing countries like India, ${ }^{1}$ whereas large bowel perforation is the most \\
common in western countries. ${ }^{2-4}$ It needs emergency surgical intervention along with adequate initial resuscitation. Several factors \\
play important role in prognosis and recovery which include timing of presentation, anatomical site, pathology of perforation, \\
extremes of age and other associated co-morbid conditions. Even with development of multidisciplinary approach and advances in \\
the management of peritonitis, mortality and morbidity due to peritonitis secondary to hollow viscous perforation remains high.
\end{tabular}

\section{MATERIALS AND METHODS}

It is a descriptive study. 78 cases with peritonitis due to hollow viscous perforation, as surgical emergencies underwent emergency laparotomy. Details of age, sex, anatomical location, signs and symptoms, reliability, complications and mortality were noted.

\section{RESULTS}

The most common age group affected is $41-60$ years. M: F ratio is 2.71: 1. Duodenal perforations are more common in the age group of 41 - 50 years and appendicular perforations are common in the age group of 21 - 40 years. Diagnosis is made clinically and confirmed by presence of pneumoperitoneum (80\%) on radiographs. All patients are subjected to emergency exploratory laparotomy along with adequate initial resuscitation. Omental patch closure of the perforation (75.64\%) is the most common procedure done. Wound infection (26.92\%) is the most common post-operative complication followed by respiratory complications (25.64\%). 8.97\% is the mortality observed in this study.

\section{CONCLUSION}

Patients suspected to be suffering from peritonitis secondary to hollow viscous perforation have to be diagnosed early and need immediate tertiary care management in the form of multimodality approach. For this, the health professionals at the primary care level have to be educated regarding early recognition and early referral to tertiary centres, so that morbidity and mortality can be minimised.

\section{KEY WORDS}

Perforation, Peritonitis, Exploratory Laparotomy.

HOW TO CITE THIS ARTICLE: Umapathi P, Babu MG. Clinical study and management of peritonitis secondary to hollow viscous perforation. J. Evolution Med. Dent. Sci. 2018;7(36):3974-3978, DOI: 10.14260/jemds/2018/888

\section{BACKGROUND}

Peritonitis due to hollow viscous perforation (Secondary) is the most common emergency encountered in general surgery practice. ${ }^{5}$ It is nothing but peritoneal inflammation due to reaction of serosal membrane of peritoneal cavity resulting from the leaked contents of the perforated viscus. It comprises majority of the emergency surgical admissions, for which emergency exploratory laparotomy procedure is

'Financial or Other Competing Interest': None.

Submission 10-08-2018, Peer Review 25-08-2018,

Acceptance 27-08-2018, Published 03-09-2018.

Corresponding Author:

Mahesh Babu G,

Civil Assistant Surgeon,

Department of General Surgery,

Apollo Institute of Medical Sciences and

Research and District Government Hospital,

Chittoor, Andhra Pradesh, India.

E-mail: maheshdrg@gmail.com uma.uwa11@gmail.com

DOI: $10.14260 /$ jemds $/ 2018 / 888$ needed in most of the patients.6,7 The diagnosis and treatment of gastrointestinal perforation remains the main problem in our country. ${ }^{8,9}$ Smoking and use of non-steroidal anti-inflammatory drugs are important risk factors for perforation. Especially, these days the inadvertent use of NSAIDS and other over-the-counter analgesics forms one of the most common risk factors.10 Perforation of the stomach, duodenum and small bowel form a considerable proportion of emergency workload than colonic perforation. ${ }^{11,5}$ The spectrum of aetiology of perforation in India differs from the western world. ${ }^{12}$ Peritonitis with septicaemia is the commonest mode of presentation.5,9 Emergency surgical intervention (Laparotomy) to control the source of infection to remove contamination by peritoneal lavage, antibiotics and physiologic support in the form of multidisciplinary approach is needed.13 The morbidity and mortality rates are high in spite of advances in perioperative care, antimicrobial therapy and intensive care support. ${ }^{14,9}$ Old age, poor general status, septic shock, delayed presentation, intervention in the form of surgery, site and pathology of perforation and peritoneal contamination are factors predicting mortality and morbidity in patients presented with peritonitis secondary to 
hollow viscous perforation. ${ }^{14}$ Perforations occur more frequently among men than women. This is due to the lifestyle and risk factors like cigarette smoking, consumption of spicy foods, alcohol abuse and physical stress. Peptic ulcer disease has decreased in men and increased in women. It is speculated that the increase in women with peptic ulcer disease was in past due to an increase in smoking and at present due to an increase in NSAID ingestion. ${ }^{15}$ Perforation can occur anywhere in the gastrointestinal tract like stomach, duodenum, jejunum, ileum, large intestine and rectum. Enteric perforations are more frequently seen in the developing countries of South East Asia, and colonic perforations in the far East. It is well known that perforations of the large bowel constitute a higher proportion of peritonitis cases in developed countries than in developing countries like India. Commonest site is gastroduodenum, where it is called peptic ulcer. Several studies revealed that peptic ulcer perforations are the commonest followed by small bowel and appendicular perforation in this region of the world.1,5 More distal the gastrointestinal visceral perforation, more is associated contamination due to increased number of organisms and more the morbidity and mortality. 16,17

Most of hollow viscous perforations are from gastroduodenal perforations. Duodenal perforation is more common than gastric perforation (Ulcer or malignancy) all over the world, the ratio ranging from $4: 1$ to $20: 1 .{ }^{18}$

Peritonitis usually presents as an acute abdomen. Local findings include abdominal tenderness, guarding, rigidity, distension and diminished bowel sounds. Systemic findings include fever, chills or rigor, tachycardia, sweating, restlessness, tachypnoea, dehydration, oliguria, disorientation and ultimately shock. The clinical manifestations of peritonitis are fluid shifts and metabolic disturbance. The heart rate and respiratory rate initially increase as a result of volumetric, intestinal, diaphragmatic, and pain reflexes. Metabolic acidosis and the increased secretion of aldosterone, antidiuretic hormone and catecholamines subsequently alter cardiac output and respiration. Protein is broken down and hepatic glycogen is mobilised as the body enters a highly catabolic state. Paralytic ileus develops, leading to profound sequestration of fluid and loss of electrolytes and protein-rich exudate. Gross abdominal distension causes diaphragmatic elevation with resultant atelectasis and pneumonia. Multiple-organ failure, coma and death will follow if peritonitis persists and fails to localise. Full blood count will demonstrate leukocytosis.

Urea and electrolytes will confirm dehydration and acute renal failure. Results are used to guide replacement of fluid and electrolytes.

Liver function tests and serum amylase- a high concentration of amylase in serum is diagnostic of acute pancreatitis, but a moderately elevated concentration can be caused by other intra-abdominal catastrophes (eg. perforated duodenal ulcer).

Arterial blood gas reflects a metabolic acidosis, often preceded by a low arterial carbon dioxide tension caused by hyperventilation.

Grouping and cross-matching- laparotomy may be indicated and therefore cross-matched blood will be required. Erect radiograph ${ }^{19}$ of the abdomen will show pneumoperitoneum in about $70-80 \%$ of visceral perforations. A left lateral decubitus radiograph of the abdomen is an alternative in those who are unable to sit up. Ultrasound may play a role in confirming or excluding specific diagnoses (e.g. subphrenic abscess). Ultrasound examination allows very rapid screening of patients in suspected patients, for triage of patients who are to undergo more invasive imaging testing. Computerised Tomography (CT) is far more accurate in negative prediction than ultrasound, and has largely replaced blind diagnostic laparotomy in the search for occult sepsis.

\section{Aims and Objectives}

1. To study the frequency of peritonitis secondary to hollow viscous perforation in relation to age, sex, anatomical location, symptoms, signs and investigations.

2. To study the surgical management of peritonitis secondary to hollow viscous perforation.

3. To study complications and outcome.

\section{MATERIALS AND METHODS}

It is a descriptive study. All patients admitted and diagnosed to have peritonitis secondary to hollow viscous perforation (treated surgically) in surgical ward of Apollo Medical College and District Government Hospital, Chittoor, during the period of August 2016 to June 2018 were included in the study.

\section{Inclusion Criteria}

All patients presenting with peritonitis secondary to hollow viscous perforation and subjected to exploratory laparotomy (based on x-ray, USG and intra-op findings).

\section{Exclusion Criteria}

Patients with peritonitis secondary to oesophageal perforation, non-gastrointestinal tract perforation, iatrogenic and traumatic perforation were excluded.

For each patient along with initial resuscitation a detailed history was noted and thorough clinical examination was performed. Emergency investigations done like haemogram, renal function tests, viral screening, serum electrolytes and ABG analysis report. Radiograph of chest and abdomen in erect position was done in all patients. This was followed by abdominal ultrasound. In some cases where the diagnostic dilemma was persistent, a CT scan of abdomen was done.

High-flow oxygen is vital for all shocked patients. Hypoxia can be monitored by pulse oximetry or measurement of arterial blood gases. Fluid resuscitation is initially with crystalloids (IV). The volume being dependent on the degree of shock and dehydration. Electrolyte (especially potassium) replacement may be required. The patient should be catheterised in order to monitor the hourly output of urine. Monitoring of central venous pressure and the use of inotropes may be appropriate in severe sepsis or in patients with comorbidity. Analgesia- opiate analgesia (IV) and an appropriate antiemetic will be required. Antibiotics should be broad-spectrum, cover aerobes and anaerobes and given intravenously. A third-generation cephalosporin and metronidazole is a common primary strategy. For patients who acquire peritonitis in hospital (e.g. anastomotic leak) or 
who require intensive care, second-line therapy with meropenem or a combination of piperacillin and tazobactam is advised. Antifungal therapy should also be considered to cover possible Candida species. Early and appropriate use of antibiotics is a key to reducing mortality in patients with septic shock associated with peritonitis. Nasogastric tube and aspiration alleviate vomiting and abdominal distension and reduces the risk of aspiration pneumonia.

Emergency exploratory laparotomy was done with standard midline incision and peritoneal cavity was explored to check all the quadrants and viscera. Standard surgical procedure (Closure with omental patch (Omentopexy), appendicectomy, primary repair, resection and anastomosis, peritoneal toilet, stoma formation) was carried out depending on anatomical site and pathology of hollow viscous perforation. A thorough warm saline wash of peritoneal cavity was carried out and drains were left in peritoneal cavity. Then abdomen was closed in layers using standard technique. Post-operatively, patients were managed with broad-spectrum antibiotic coverage along with maintenance of fluid and electrolyte balance with necessary investigations till discharge from hospital. Immediate postoperative complications were identified and treated timely. All discharged patients were reviewed in OPD at $2^{\text {nd }}$ week, $1^{\text {st }}$ month and $3^{\text {rd }}$ month. Relevant data were noted whenever needed. Mortality in this study refers to death of patients in the hospital during same admission as the episode of peritonitis.

Most common complications are wound infection. Intraabdominal collection, burst abdomen, ARDS and pneumonia, faecal fistula, anastomotic leak, bacteraemic/ endotoxic shock, systemic inflammatory response syndrome, paralytic ileus, portal pyaemia/ liver abscess, multiorgan dysfunction syndrome and death. (Adhesional small bowel obstruction seen in later periods). Factors affecting mortality and complications are late presentation, site of perforation, advanced age, delay in treatment, septicaemia, multiorgan failure and other comorbidities.

\section{RESULTS}

- The most common age group affected is 41 - 60 years. $\mathrm{M}: \mathrm{F}$ ratio is 2.71: 1 .

- Duodenal perforations are more common in the age group of 41 - 50 years and appendicular perforations are common in the age group of 21 - 40 years.

- Most of these patients present with pain abdomen associated with abdominal distension, vomiting and fever. Guarding and rigidity is the most common sign elicited followed by absent bowel sounds.

- Diagnosis is made clinically and confirmed by presence of pneumoperitoneum (80\%) on radiographs.

- All patients are subjected to emergency exploratory laparotomy along with adequate initial resuscitation. Omental patch closure of the perforation $(75.64 \%)$ is the most common procedure done.

- Wound infection (26.92\%) is the most common postoperative complication followed by respiratory complications (25.64\%).

- $\quad 8.97 \%$ is the mortality observed in this study.

\section{DISCUSSION}

\begin{tabular}{|c|c|c|c|}
\hline Age Group in Years & Male (\%) & Female (\%) & Total (\%) \\
\hline $0-20$ & $4(5.1 \%)$ & $2(2.5 \%)$ & $6(7.6 \%)$ \\
\hline $21-40$ & $21(26.9 \%)$ & $7(8.9 \%)$ & $28(35.8)$ \\
\hline $41-60$ & $24(30.7 \%)$ & $8(10.2 \%)$ & $32(41 \%)$ \\
\hline$>60$ & $8(10.2 \%)$ & $4(5.1 \%)$ & $12(15.3)$ \\
\hline Total & $\mathbf{5 7 ( 7 3 \% )}$ & $\mathbf{2 1}(\mathbf{2 7 \% )}$ & $\mathbf{7 8 ( 1 0 0 \% )}$ \\
\hline \multicolumn{4}{|c}{ Table 1. Age and Sex Incidence } \\
\hline
\end{tabular}

From the above table, it was observed that the most common affected people are males in the present study. Out of total 78 patients, $57(73.07 \%)$ were males and 21 $(26.93 \%)$ were females. Male preponderance was seen in almost all anatomical variations. 42 males and 14 females had duodenal perforation. Appendicular perforation was seen in 7 males and 3 females. Ileal perforation was present in 5 males and 2 females, only 1 male patient had perforated large bowel (Sigmoid diverticulum). Gastric perforation was seen in 2 male and 2 female patients.

The most common age group affected in the present study belongs to $4^{\text {th }}$ and $5^{\text {th }}$ decades, followed by $2^{\text {nd }}$ and $3^{\text {rd }}$ decades. Duodenal perforation is seen mostly in $4^{\text {th }}$ and $5^{\text {th }}$ decades. Appendicular perforation is more in $2^{\text {nd }}$ and 3 rd decades. Similar results were obtained in studies conducted by Pooja Batra et al, ML Ramachandra et al and Nitin Agarwal et al.

\begin{tabular}{|c|c|c|}
\hline Clinical Feature & Frequency & Percentage \\
\hline Pain abdomen & 78 & $100 \%$ \\
\hline Nausea and vomiting & 44 & $56.41 \%$ \\
\hline Fever & 60 & $76.92 \%$ \\
\hline Distension & 63 & $80.76 \%$ \\
\hline Guarding and rigidity & 71 & $91.02 \%$ \\
\hline Pneumoperitoneum & 63 & $80.76 \%$ \\
\hline Shock Table 2. Clinical Features \\
\hline \multicolumn{2}{|c|}{} \\
\hline
\end{tabular}

It was observed from the above table that the most common clinical presentation was pain abdomen. All 78 $(100 \%)$ patients presented with pain abdomen. Abdominal distension was found in $63(80.76 \%)$ patients. Fever was seen in $60(76.92 \%)$ patients. Nausea and vomiting were present in $44(56.41 \%)$ patients. Rigidity and guarding was the most common sign found in 71 (91.02\%) patients and bowel sounds were absent in 65 patients. Gas under the right dome of diaphragm (Pneumoperitoneum) was seen on x-ray erect abdomen of $63(80.76 \%)$ patients. Most unfavourable presentation was patients with established shock, 4 patients presented with this. The results were comparable with studies conducted by Rajender Singh Jhobta et al,5 ML Ramachandra et $\mathrm{al}^{20}$ and Batra et $\mathrm{al}^{21}$

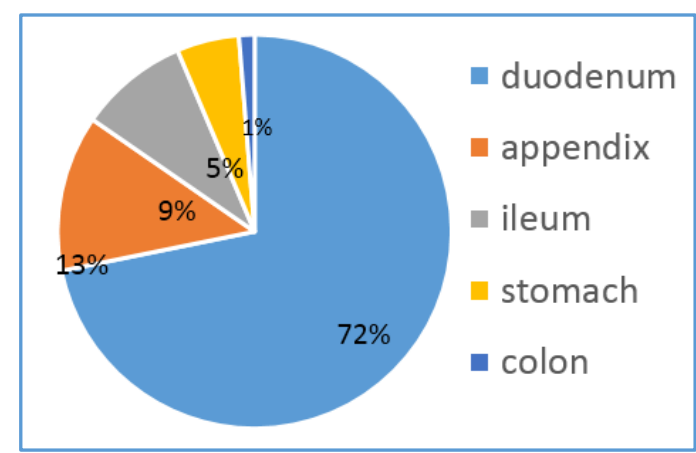

Figure 1. Anatomical Site Distribution 
Majority of the perforations observed in this study were seen in duodenum followed by appendix, ileum stomach and colon respectively as shown in the Figure. Similar results were obtained in studies conducted by ML Ramachandra et al and Pooja Batra et al.

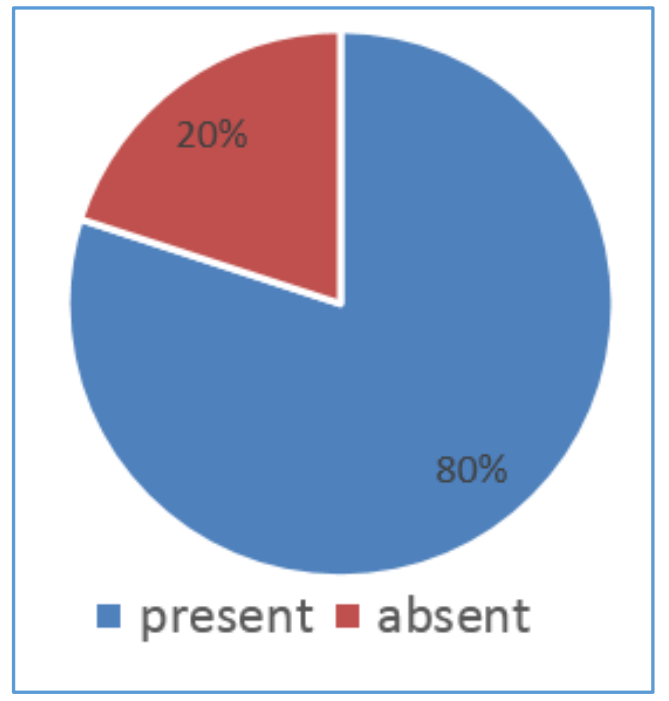

Figure 2. Presence of Pneumoperitoneum

Pneumoperitoneum was observed in $80 \%$ of the cases in this study.

\begin{tabular}{|c|c|c|}
\hline Procedure & Frequency & Percentage \\
\hline Omental Patch Closure & 59 & $75.64 \%$ \\
\hline Open Appendicectomy & 10 & $12.82 \%$ \\
\hline Simple Closure & 3 & $3.84 \%$ \\
\hline $\begin{array}{l}\text { Resection and } \\
\text { Anastomosis }\end{array}$ & 3 & $3.84 \%$ \\
\hline Resection and Stoma & 3 & $3.84 \%$ \\
\hline Total & 78 & $100 \%$ \\
\hline \multicolumn{3}{|c|}{ Table 3. Types of Surgical Procedure } \\
\hline
\end{tabular}

Graham's Omental patch closure is the commonest procedure in this study, done in 59 (75.64\%) patients. For all appendicular perforations (10 patients), open appendicectomy procedure was done. Resection of bowel and primary anastomosis was done in patients with single ileal perforation. Results obtained were inconsistent with studies conducted by Rajeshwara KV et al. ${ }^{22}$

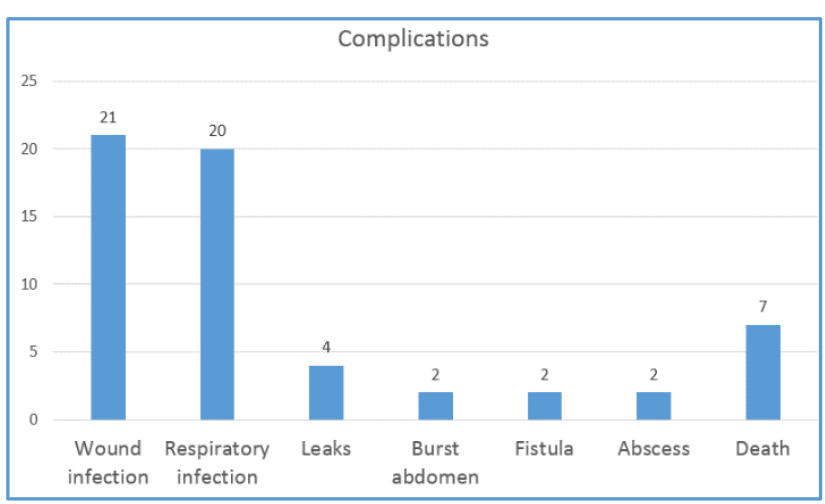

Figure 3. Distribution of Complications

Most common complication observed in the present study was wound infection seen in $21(26.92 \%)$ patients. All these patients were managed with culture sensitive antibiotics. Four patients among these needed secondary suturing. Respiratory complications (Pneumonia, abscess, respiratory failure, ARDS) were also most common in the present series, which occurred in 20 (25.64\%) patients. Post-operative leak was noticed in $4(5.12 \%)$ patients. Similar results were obtained in studies conducted by Ramachandra et al and Pooja Batra et al. Burst abdomen was seen in $2(2.56 \%)$ patients. Enterocutaneous fistula was seen in $2(2.56 \%)$ patients. Pelvic abscess was seen in $2(2.56 \%)$ patients. One patient of duodenal perforation was found to have sub-acute intestinal obstruction.

\section{CONCLUSION}

Patients suspected to be suffering from peritonitis secondary to hollow viscous perforation have to be diagnosed early and need immediate tertiary care management in the form of multimodality approach. For this, the health professionals at the primary care level have to be educated regarding early recognition and early referral to tertiary centres, so that morbidity and mortality can be minimised.

\section{REFERENCES}

[1] Agarwal N, Saha S, Srivastava A, et al. Peritonitis: 10 years' experience in a single surgical unit. Trop Gastroenterol 2007;28(3):117-20.

[2] Crawford E, Ellis H. Generalised peritonitis - the changing spectrum. A report of 100 consecutive cases. Brit J Clin Pract 1985;39(5):177-8.

[3] Washington BC, Villalba MR, Lauter CB, et al. Cefamandole-erythromycin-heparin peritoneal irrigation: an adjunct to the surgical treatment of diffuse bacterial peritonitis. Surgery 1983;94(4):57681.

[4] Nishida T, Fujita N, Megawa T, et al. Post-operative hyperbilirubinemia after surgery for gastrointestinal perforation. Surg Today 2002;32(8):679-84.

[5] Jhobta RS, Attri AK, Kaushik R, et al. A spectrum of perforation peritonitis in India - review of 504 consecutive cases. World J Emerg Surg 2006;1:26.

[6] Donovan AJ, Berne TV, Donovan JA. Perforated duodenal ulcer - an alternative therapeutic plan. Arch Surg 1998;133(11):1166-71.

[7] Beniwal US, Jindal D, Sharma J, et al. Comparative study of operative procedures in typhoid perforations. Ind J Surg 2003;65(2):172-6.

[8] Dorairajan LN, Gupta S, Deo SV, et al. Peritonitis in India - a decade's experience. Trop Gastroenterol 1995;16(1):33-8.

[9] Gupta S, Kaushik R. Peritonitis - the Eastern experience. World J Emerg Surg 2006;1:13.

[10] Fries JF, Miller SR, Spitz PW, et al. Toward an epidemiology of gastropathy associated with nonsteroidal anti-inflammatory drug use. Gastroenterology 1989;96(2 Pt 2 Suppl):647-55.

[11] Janikalpesh, Saxena AK. Management of large sized duodenal ulcer perforation by omental plugging. A new technique a prospective study of 100 patients. Ind J Surg 2000;62(2):134-8.

[12] Ersumo T, W/Meskel Y, Kotisso B. Perforated peptic ulcer in Tikur Anbessa hospital: a review of 74 cases. Ethiop Med J 2005;43(1):9-13. 


\section{Jemds.com}

[13] Schein M. Surgical management of intra-abdominal infection: is there any evidence? Langenbecks Archives of Surgery 2002;387(1):1-7.

[14] Solomkin JS, Wittman DW, West MA, et al. Intraabdominal infections. In: Schwartz SI, Shires GT, Spencer FC, et al. eds. Principles of Surgery. $7^{\text {th }}$ edn. New York, USA: McGraw-Hill 1999;2:1515-51.

[15] Danapat MC, Mukherjee LM, Mishra SB, et al. Gastrointestinal perforations. Indian Journal of Surgery 1991;53(5):189-93.

[16] Khan S, Khan IU, Aslam S, et al. Retrospective analysis of abdominal surgeries at Nepalgunj Medical College (NGMC), Nepalgunj, Nepal: 2 years of experience. Kathmandu Univ Med J (KUMJ) 2004;2(4):336-43.

[17] Yadav SS, Srinarayan. An experience with cases of peritonitis at Bheri Zonal hospital, Nepalgunj. J Soc Surg Nepal 2002;5:33-6.

\section{Original Research Article}

[18] Siu WT, Leong HT, Law BK, et al. Laparoscopic repair for perforated peptic ulcer: a randomized controlled trial. Ann Surg 2002;235(3):313-9.

[19] Lee MJ. Non-traumatic abdominal emergencies: imaging and intervention in sepsis. European Radiology 2002;12(9):2172-9.

[20] Ramachandra ML, Jagadesh B, Chandra SBC. Clinical study and management of secondary peritonitis due to perforated hollow viscous. Arch Med Sci 2007;3:61-8.

[21] Batra P, Gupta D, Narang R, et al. Spectrum of gastro intestinal perforation peritonitis in Rural Central India. J MGIMS 2013;18(1):44-8.

[22] Rajeshwara KV, Praveen KK, Rao PK, et al. Spectrum of secondary peritonitis due to hollow viscus perforation in a tertiary care hospital. Journal of Evolution of Medical and Dental Sciences 2014;3(27). 